\title{
corseq: fast and efficient identification of favoured codons from next generation sequencing reads
}

\author{
Salvatore Camiolo ${ }^{\text {Corresp., }}{ }^{1}$, Andrea Porceddu ${ }^{1}$ \\ 1 Dipartimento di Agraria, Università degli studi di Sassari, Sassari, Italy \\ Corresponding Author: Salvatore Camiolo \\ Email address: scamiolo@uniss.it
}

Background:Optimization of transgene expression can be achieved by designing coding sequences with the synonymous codon usage of genes which are highly expressed in the host organism. The identification of the so called "favoured codons" generally requires the access to either the genome or the coding sequences and the availability of expression data. Results:Here we describe corseq, a fast and reliable software for detecting the favoured codons directly from RNAseq data without prior knowledge of genomic sequence or gene annotation. The presented tool allows the inference of codons that are preferentially used in highly expressed genes while estimating the transcripts abundance by a new kmer based approach. corseq is implemented in Python and runs under any operating system. The software requires the Biopython 1.65 library (or later versions) and is is available under the 'GNU General Public License version 3' at the project webpage https://sourceforge.net/projects/corseq/files Conclusion:corseq represents a faster and easy-to-use alternative for the detection of favoured codons in non model organisms. 
1 corseq: fast and efficient identification of favoured codons from next generation sequencing

2 reads.

3 Salvatore Camiolo, Andrea Porceddu

4 Dipartimento di Agraria, Università degli Studi di Sassari, Viale Italia 39, 07100, Sassari, Italy

7 Corresponding author

8 Salvatore Camiolo

9 Dipartimento di Agraria, Università degli Studi di Sassari, Viale Italia 39, 07100, Sassari, Italy

10 Email address: scamiolo@uniss.it

11

12

13

14

15

16

17

18

19

20

21

22 


\section{Abstract}

25 Background: Optimization of transgene expression can be achieved by designing coding

26 sequences with the synonymous codon usage of genes which are highly expressed in the host

27 organism. The identification of the so called "favoured codons" generally requires the access to

28 either the genome or the coding sequences and the availability of expression data.

29 Results: Here we describe corseq, a fast and reliable software for detecting the favoured codons

30 directly from RNAseq data without prior knowledge of genomic sequence or gene annotation.

31 The presented tool allows the inference of codons that are preferentially used in highly expressed

32 genes while estimating the transcripts abundance by a new kmer based approach. corseq is

33 implemented in Python and runs under any operating system. The software requires the

34 Biopython 1.65 library (or later versions) and is is available under the 'GNU General Public

35 License version 3' at the project webpage https://sourceforge.net/projects/corseq/files

36 Conclusion: corseq represents a faster and easy-to-use alternative for the detection of favoured

37 codons in non model organisms. 


\section{Introduction}

50 The preferential usage of a specific synonymous codons set, also referred to as codon bias, was observed for a variety of organisms as diverse as yeasts, plants, bacteria and metazoan (Plotkin \& Kudla, 2011). Codons that mirror the most abundant tRNA (known as favoured codons) can speed up the translational machinery and are expected, for this reason, to be preferred by natural selection in highly expressed genes (Bulmer, 1991). Knowledge of favoured codons for a host organism is a prerequisite for the design of transcripts that are efficiently translated (Quax et al., 2015). Such a goal may be achieved by performing a relative synonymous codon usage analysis of the correspondences (RSCU-COA, as implemented in the widely used software codonw (Peden, 2000), codonw.sourceforge.net/) . This approach relies on the assumption (not always verified) that codon bias and expression level are strongly correlated. Alternatively, favoured codons can be detected by directly comparing the codon usage of highly and lowly expressed genes (Chiapello et al., 1998). This methodology requires both the genic sequences and their expression profile. If a suitable annotation is not available (e.g. for a new non sequenced organism) transcripts may be reconstructed by mapping RNAseq sequence data on a reference genome or by direct de novo assembly, both approaches representing a time consuming and hardware demanding task (Conesa et al., 2016). Here we propose corseq, a user friendly, fast and reliable tool for the direct identification of the favoured codons from RNAseq data without prior knowledge of genomic/genic sequence or gene expression data. The presented software aims to identify the putative open reading frame (ORF) within the sequences generated by an RNAseq experiment and quantify the relative codons frequency. corseq uses a nucleotide kmer analysis to 
70 infer those codons that are preferentially used in highly abundant transcripts by considering the

71 kmer frequency as a proxy of the expression value.

73 Material and methods

74 Sequences and expression data

75 Coding sequences for the two plants Arabidopsis thaliana and Oryza sativa where downloaded

76 by the Phytozome 11 database, for the four bacteria Escherichia coli, Staphylococcus aureus,

77 Lactobacillus pentosus, Bacillus thuringiensis were obtained from the EMBL bacteria database,

78 for the two fungi Saccharomyces cerevisiae, Fusarium graminearum were downloaded from the

79 EMBL fungi database, and for the three metazoan Mus musculus, Drosophila melanogaster,

80 Caenorhabditis elegans were obtained from the EMBL metazoan database (see table 1). For the

81 same species, RNAseq data were downloaded from the NCBI SRA database (see table 1 for the

82 corresponding accession numbers).

\section{Software implementation}

84 corseq is implemented in Python and can run under any operating system. The software

85 (available at https://sourceforge.net/projects/corseq/files) does not necessitate any installation

86 and only requires the library Biopython 1.65 (Cock et al., 2009) or later version. Corseq takes in

87 input an RNAseq fastq formatted file together with a multifasta protein training set. The software

88 already includes general training sets for viridiplantae, fungi, bacteria and metazoan although

89 user defined proteins can be added. Provided training sets comprise the Uniprot proteins

90 (www.uniprot.org) filtered for the kingdoms viridiplantae (38,739 sequences), fungi (32,674

91 sequences), bacteria (333,047 sequences) and metazoan (105,209 sequences). The corseq

92 pipeline starts with the computation of all the amino acids kmers present in the selected protein 
93 training set (the flag kp allows the selection of the kmer size at this stage). The identified

94 sequences are stored into a "Peptide set" (Figure 1). Here, all the presented results involve the

95 use of 11 amino acids kmers, but this parameter can be changed by the user. From the selected

96 RNAseq file a user defined number of bases (NOB) are extracted and used to perform the

97 downstream analysis. To do so, the needed number of reads is randomly selected from the

98 original dataset. Each of the selected reads is then searched in both forward and reverse

99 orientations for nucleotides 33 -mers (e.g. $3 * \mathrm{kp}$ ) that codes for any of the 11-mer amino acid

100 sequences present in the Peptide set. When a match is found, the corresponding position and

101 orientation are considered indicative of the original transcript correct frame. The reads are thus

102 cut accordingly to the found position/orientation and stored in what we called as a pseudo-ORF

103 set (Figure 1). For each of the found pseudo-ORFs, the enclosed nucleotide kmers are counted by

104 performing a sliding window analysis toward the sequence with a window size equal to user

105 defined value (flag $-\mathrm{k}$ in the corseq options) and a window step equal to 3 . Extremely recurrent

106 kmers are considered as portions of highly expressed genes and, vice versa, kmer with few

107 occurrences are more likely to derive from lowly expressed genes. All kmers are expected to

108 contain (or to be enriched in) in frame codons sequences due to the method used for the

109 generation of the pseudo-ORF and to the selected window step $=3$. After sorting the kmers in

110 ascending frequency order, the $2 \%$ most frequent and the $2 \%$ less frequent sequences are

111 selected. A Chi-squared contingency test analysis is then performed for each codon between

112 these two groups. For each codon, $2 \times 2$ contingency tables are filled according the following

113 scheme: the first row reports the number of occurrences of the codon and the number of

114 occurrences of its synonymous peers in high frequency kmers while the second row reports the

115 same values found in the low frequency kmers (Zhou, Weems \& Wilke, 2009). An odds ratio 
116 and a significance value are generated so that odds ratio $>1$ with a significance level $>3.84(\mathrm{p}<$

1170.05 ) are considered indicative of optimality (see supplemental files S1 for a more detailed

118 description of the algorithm and the user definable parameters). The effect of the kmer frequency

119 percentage threshold (corseq default value $=2 \%$ ) was investigated and consistent results were

120 obtained when this value ranged between $1 \%$ and $15 \%$ (supplemental File S2).

122

\section{Conventional expression method}

124 For each analysed species a RNAseq experiment together with transcripts and coding sequences

125 were downloaded from publicly available databases (Table 1). Favoured codons were first

126 identified by comparison of codon usage profiles between the most extremely highly and lowly

127 expressed coding sequences, an approach that has proven to be an effective means to reveal

128 preferences in codon use that are linked to translation (Shields et al., 1988; Duret \& Mouchiroud,

129 1999; Ingvarsson, 2008; Cutter, 2008; Wang et al., 2011; Whittle \& Extavour, 2015). Hereafter

130 we refer to this method as the conventional expression method (CEM). Briefly, RNAseq

131 sequences were aligned to the coding sequences with bwa (Li \& Durbin, 2010) (default

132 parameters) and transcript abundance were quantified by the tool express (Roberts \& Pachter,

133 2013) (default parameters). We then used a chi square test to compare the codon usage of the

$1345 \%$ most highly and most lowly expressed transcripts, i.e. a method that is implemented in the

135 software Seforta (Camiolo et al., 2014). At this stage, $2 \times 2$ contingency tables were created

136 similar to those discussed in the previous section.

137

138 Results 


\section{Result consistency among replicates}

140 Since corseq randomly selects a user defined number of bases (NOB) from the original RNAseq

141 dataset to run the analysis on, we wondered whether the identified favoured codons were

142 consistent when repeating the analysis several times. Moreover, we checked whether possible

143 fluctuations were associated to the selected number of bases. For this reason we carried out 5

144 replicates of each of the following NOB values: $5 \mathrm{Mb}, 10 \mathrm{Mb}, 20 \mathrm{Mb}$ and $40 \mathrm{Mb}$. Such a test

145 was performed on A. thaliana, S. cerevisiae, E. coli, M. musculus, and D. melanogaster. Our

146 results show only few non concordant favoured codons with this trend being further minimized,

147 as expected, for higher NOB values (Figure 2). For this reason, the corseq default value for the

148 selected number of bases is set to 20 millions.

149 The effect of the nucleotide kmer size

150 Estimation of highly and lowly expressed genes is performed by computing high and low

151 representative k-mers in the pseudo-ORFs (see Material and methods section). We tested the

152 effect of the k-mer size in detecting the favoured codons. Hence, we performed 5 replicates for 4

153 k-mer sizes (12mer, 21mer, 30mer, 39mer) and for 5 species (A. thaliana, S. cerevisiae, E. coli,

154 M. musculus, and D. melanogaster). As reported in Figure 3 few differences were observed

155 among the tested k-mer sizes. However, since lower values may possibly be associated to an

156 increased chance that some k-mer is shared between highly and lowly expressed genes we

157 decided to leave 39 mer as the default k-mer size for corseq. This value also proved to slightly

158 improve the number of favoured codons that are called concordantly with the conventional

159 expression method (CEM, Figure 4).

160 The effect of the provided number of reads. 
161 The software performance, as compared to the CEM, was estimated by using large RNAseq

162 dataset $(9,955,999$ reads, sequence length $=150 \mathrm{nt})$. We wondered how the detection of the

163 favoured codons may be influenced by reducing the initial dataset size. Thus, we randomly

164 selected a number of reads from the Arabidopsis thaliana RNAseq file ranging from as low as

16525,000 reads (accounting for 3,75 Mb) up to a maximum of 2,000,000 reads (accounting for 300

$166 \mathrm{Mb}$ ). We then ran corseq by collecting $20 \mathrm{Mb}$ with a nucleotide k-mer size of 39 . Notably,

167 although 25,000 reads are not sufficient to fulfil the requested 20 million bases, a suitable dataset

168 is nevertheless created since extraction of random reads is performed with replacement (e.g. the

169 final dataset is more likely populated by reads deriving from the most abundant transcripts). The

170 performance was estimated by calculating a Pearson correlation coefficient between the odds

171 ratio of the codons calculated by both corseq and CEM with the latter being carried out on the

172 original RNAseq dataset. As expected, the calculated correlation increased at higher dataset sizes

173 up to the value of 125,000 reads (accounting for $18,75 \mathrm{Mb}$ ) when a plateau was reached.

174 Notably, even for the smallest RNAseq dataset the odds ratio correlation proved to be higher

175 than 0.8 (Figure 5a). Size of the analysed RNAseq file represents the primary determinant of the

176 run times. In this regard, corseq was able to run the described analyses in a time range comprised

177 between 2.1 and 12.9 minutes (Figure 5b) while the analysis on the entire RNAseq file took as

178 low as 42.5 minutes to complete.

179 Performance benchmark

180 Favoured codons were calculated by using corseq with default parameters (kmer size for the

181 proteins training set $=11$, nucleotide $\mathrm{k}-$ mer size for the pseudo-ORF $=39$, number of collected

182 bases $=20$ millions) for the 12 species reported in Table 1 . The same analysis was performed

183 with the CEM as described in the main manuscript. The two outcomes were compared by 
184 considering the following parameters: (a) the number of concordant favoured codons, (b) the

185 number of method specific favoured codons; (c) the percentage of codons for which the odds

186 ratio featured the same direction with the two methods (odds ratio $>1$ for both or odds ratio $<1$

187 for both); (d) the Pearson correlation between the odds ratio calculated with the two methods; (e)

188 the amino acid consensus (AAC) that is the percentage of amino acids for which at least one of

189 the called favoured codon (e.g. more than one synonymous codon may be called as favourite) is

190 concordant between the two methods. The results are reported in Table 2.

\section{Discussion}

192 Optimizing the coding sequence of a heterologues transcript is the first step to maximize its 193 expression in a host organism. This process is primarily performed by designing a coding

194 sequence featuring the same codons that are used in host highly expressed genes, e.g. its

195 favoured codons. Notably, such an approach does not rule out the importance of sub-optimal (or

196 even rare) codons in increasing the expression of a heterologues gene (Shah \& Gilchrist, 2011;

197 Porceddu, Zenoni \& Camiolo, 2013; Mauro \& Chappell, 2014; Gilchrist et al., 2015; Camiolo,

198 Sablok \& Porceddu, 2017), since it is known that additional determinants may play a primary

199 role as, for example, the protein co-translational folding (Yang, Chen \& Zhang, 2014) and the

200 mRNA stability (Wu et al., 2004).

201 Here we present corseq, a new bioinformatics tools capable of quickly computing the favoured

202 codons of an organism by analysing RNAseq data. The software allows for the determination of

203 the codons that are used in the highly expressed transcripts but does not imply an actual

204 quantification of the gene expression. Instead, corseq aims to identify position and orientation of

205 in frame exons (or portions of them) within the RNAseq reads and computes the codons that are

206 more likely to occur in the most abundant transcripts as a result of a combination of kmer 
207 analysis and sliding window approach (see Material and methods section). First, corseq

208 randomly samples the provided RNAseq fastq file in order to work only on a data subset and

209 thus reduce the run time. Our tests proved that sampling as low as 20 millions bases allows to

210 minimize the risk of calling different favoured codons sets among replicates (Figure 2). This

211 number also ensures the maximum concordance with the favoured codons found with alternative

212 methods (e.g. the conventional expression method, Figure 5). Then, the software extracts from

213 each read a pseudo-ORF by inferring its position and orientation from the homology of their

214 coded peptides as compared to a reference protein database (Figure 1). From each pseudo-ORF

215 all possible kmers are computed with the most recurrent being considered as belonging to highly

216 expressed genes. The effect of the k-mer size was investigated and a good agreement among all

217 the analysed values was found (Figure 4).

218 We benchmarked corseq versus one of the most common methods for the identification of

219 favoured codons, herein referred to as the conventional expression method (see Material and

220 methods section). While a good agreement was found between the two approaches (Table 2), few

221 differences emerged that deserve a careful investigation. Indeed, codons that are found only by

222 corseq should not be necessarily treated as false positive. In fact, corseq generally achieves

223 higher significance levels (due to the increased number of comparisons) as compared to the CEM

224 which may increase its sensitivity in calling as favoured those codons that are only slightly over

225 used in highly expressed genes or whose coded amino acid is poorly represented in the proteome.

226 As a way of example in Arabidopsis thaliana the cysteine codon TGC is called as favoured by

227 corseq but not by the CEM. This may be possibly due to the cysteine being one of the less

228 frequent amino acids in plants and the identification of its favoured codons can be difficult if a

229 higher number of genes is not analysed. In the effort of understanding whether TGC is a false 
230 positive or, on the contrary, is the result of the high corseq sensitivity we investigated the

231 genomic tRNA database (Chan \& Lowe, 2016) and found that the anticodon for TGC is indeed

232 the most abundant. Similarly, in Arabidopsis ACC test failed to find a concordance in the called

233 favoured codons for the amino acid Proline with corseq calling CCA and the CEM calling CCT

234 with the former actually featuring the highest number of anticodon tRNA in this plant species.

235 This phenomenon was observed for several other codons in several other species (e.g. TGC and 236 GAC in L. pentosus, GAG in B. thuringensis, CAA in C. elegans).

237 All the odds ratio and significance values for the comparisons described in this benchmark are 238 reported in Supplemental file S3

\section{Conclusions}

241 The described software is a valuable tool for the identification of the favoured codons for

242 organisms with neither genomic nor annotation data available. We tested corseq on several 243 organisms spanning from plants to bacteria, fungi and metazoan and found, overall, favoured

244 codon sets featuring high agreement with those calculated by the conventional expression

245 method. Indeed, a 0.85 average correlation was observed between the Chi-squared odds ratio

246 found with the two methods whereas the percentage of favoured codons detected by corseq and

247 concordant with the CEM ranged from 75 to $100 \%$. Finally, most of the favoured codons

248 identified by corseq were also previously detected by the mean of alternative methods in $S$.

249 cerevisiae, E. coli, M. musculus, D. melanogaster (Zhou, Weems \& Wilke, 2009), A. thaliana,

250 O. sativa (Camiolo, Sablok \& Porceddu, 2017), S. aureus

251 (http://codonw.sourceforge.net/JohnPedenThesisPressOpt_water.pdf), C. albicans (Lloyd \&

252 Sharp, 1992) and C. elegans (Riddle et al., 1997) 
253 Corseq is fast (in our tests the running time ranged from few minutes to around three hours for

254 the largest fastq file), reliable and, at the best of our knowledge, it represents the only alternative 255 to the much more complex methodology involving transcript de novo assembly/annotation/reads 256 alignment/expression quantification/favoured codon computation.

257

258 Acknowledgement

259 We would like to thank Dr. Laura Mula and Dr. Emanuela Spanu for their useful suggestions at 260 the initial stage of the software development.

261

262

263

264

265

266

267

268

269

270

271

272 Figures legends

273 Figure 1: The corseq pipeline. (a) Population of the "peptide set"; selected proteins (suitable for

274 the analysed species) are subjected to a kmer analysis using a sliding window approach, thus

275 producing a set of amino acid kmers. (b) Identification of the pseudo-ORFs; RNAseq reads are 
276 sampled at each position by using a sliding window approach; the produced subsequences are

277 translated and, if stop codons are not present, the corresponding pseudo protein is compared with

278 the set of previously identified amino acid kmers. (c) Quantification of the nucleotide kmers

279 generated by the pseudo-ORFs; sequences for which the corresponding protein matched an

280 amino acid kmer, are split in their kmers that are thus quantified. (d) favoured codons

281 computation; high and low frequency kmers are considered as proxy of high and low expression

282 genes respectively; favoured codons are then computed by comparing, for each amino acid, the

283 usage of the synonymous codons between these two sequences datasets.

284 Figure 2: Venn diagrams showing the number of detected favoured codons for 5 replicates, 4

285 values of number of bases and 5 species. For each presented diagram, each colour region reports

286 the number of codons that were found to be favoured following a corseq run. Overlapping

287 regions reports the number of concordant favoured codons between 5 replicates for the specified

288 combination of species / number of bases.

289

290 Figure 3: Venn diagrams showing the number of detected favoured codons for 5 replicates, 4

291 nucleotide kmer sizes and 5 species. For each presented diagram, each colour region reports the

292 number of codons that were found to be favoured following a corseq run. Overlapping regions

293 reports the number of concordant favoured codons between 5 replicates for the specified

294 combination of species / nucleotide kmer size.

295

296 Figure 4: Venn diagrams showing the number of detected favoured codons that are shared

297 between corseq with 4 different k-mer sizes and the conventional expression method. For each

298 presented diagram, each colour region reports the number of codons that were found to be

299 favoured following a corseq run. Overlapping regions reports the number of concordant favoured

300 codons between 4 replicates and the conventional expression method for the specified species.

301

302 Figure 5: (a) Pearson correlation between the codons odds ratio calculated with corseq and the

303 classical expression method. For each point the corseq odds ratio values were calculated starting 
304 from RNAseq dataset of different size. (b) Run times of the corseq elaboration for RNAseq

305 dataset of different size.

306

307 References

308

309

310

311

312

313

314

315

316

317

318

319

320

321

322

323

324

325

326

327

328

329

330

331

332

333

334

335

336

337

338

339

340

341

342

343

344

345

346

Bulmer M 1991. The selection-mutation-drift theory of synonymous codon usage. Genetics 129:897-907.

Camiolo S, Melito S, Milia G, Porceddu A 2014. Seforta, an integrated tool for detecting the signature of selection in coding sequences. BMC research notes 7:240. DOI: 10.1186/17560500-7-240.

Camiolo S, Sablok G, Porceddu A 2017. The Evolutionary Basis of Translational Accuracy in Plants. G3 (Bethesda, Md.) 7:g3.117.040626-2373. DOI: 10.1534/g3.117.040626.

Chan PP, Lowe TM 2016. GtRNAdb 2.0: an expanded database of transfer RNA genes identified in complete and draft genomes. Nucleic Acids Research 44:D184-D189. DOI: 10.1093/nar/gkv1309.

Chiapello H, Lisacek F, Caboche M, Hénaut A 1998. Codon usage and gene function are related in sequences of Arabidopsis thaliana. Gene 209:GC1-GC38.

Cock PJA, Antao T, Chang JT, Chapman BA, Cox CJ, Dalke A, Friedberg I, Hamelryck T, Kauff F, Wilczynski B, de Hoon MJL 2009. Biopython: freely available Python tools for computational molecular biology and bioinformatics. Bioinformatics (Oxford, England) 25:1422-1423. DOI: 10.1093/bioinformatics/btp163.

Conesa A, Madrigal P, Tarazona S, Gomez-Cabrero D, Cervera A, McPherson A, Szcześniak MW, Gaffney DJ, Elo LL, Zhang X, Mortazavi A 2016. A survey of best practices for RNAseq data analysis. Genome biology 17:13. DOI: 10.1186/s13059-016-0881-8.

Cutter AD 2008. Divergence Times in Caenorhabditis and Drosophila Inferred from Direct Estimates of the Neutral Mutation Rate. Molecular Biology and Evolution 25:778-786. DOI: $10.1093 / \mathrm{molbev} / \mathrm{msn} 024$.

Duret L, Mouchiroud D 1999. Expression pattern and, surprisingly, gene length shape codon usage in Caenorhabditis, Drosophila, and Arabidopsis. Proceedings of the National Academy of Sciences 96:4482-4487. DOI: 10.1073/pnas.96.8.4482.

Gilchrist MA, Chen W-C, Shah P, Landerer CL, Zaretzki R 2015. Estimating Gene Expression and Codon-Specific Translational Efficiencies, Mutation Biases, and Selection Coefficients from Genomic Data Alone. Genome biology and evolution 7:1559-1579. DOI: $10.1093 / \mathrm{gbe} / \mathrm{evv} 087$.

Ingvarsson PK 2008. Molecular evolution of synonymous codon usage in Populus. BMC Evolutionary Biology 8:307-13. DOI: 10.1186/1471-2148-8-307.

Li H, Durbin R 2010. Fast and accurate long-read alignment with Burrows-Wheeler transform. Bioinformatics (Oxford, England) 26:589-595. DOI: 10.1093/bioinformatics/btp698.

Lloyd AT, Sharp PM 1992. Evolution of codon usage patterns: the extent and nature of divergence between Candida albicans and Saccharomyces cerevisiae. Nucleic Acids Research 20:5289-5295.

Mauro VP, Chappell SA 2014. A critical analysis of codon optimization in human therapeutics. Trends in Molecular Medicine 20:604-613. DOI: 10.1016/j.molmed.2014.09.003.

Peden JF 2000. Analysis of codon usage. PhD thesis, University of Nottingham 
347 Plotkin JB, Kudla G 2011. Synonymous but not the same: the causes and consequences of codon bias. 12:32-42. DOI: $10.1038 / \mathrm{nrg} 2899$.

349

350

351

352

353

354

355

356

357

358

359

360

361

362

363

364

365

366

367

368

369

370

371

372

373

374

375

376

377

378

379

Porceddu A, Zenoni S, Camiolo S 2013. The signatures of selection for translational accuracy in plant genes. Genome biology and evolution 5:1117-1126. DOI: 10.1093/gbe/evt078.

Quax TEF, Claassens NJ, Söll D, van der Oost J 2015. Codon Bias as a Means to Fine-Tune Gene Expression. Molecular cell 59:149-161. DOI: 10.1016/j.molcel.2015.05.035.

Riddle DL, Blumenthal T, Meyer BJ, Priess JR 1997. C. elegans II.

Roberts A, Pachter L 2013. Streaming fragment assignment for real-time analysis of sequencing experiments. Nature methods 10:71-73. DOI: 10.1038/nmeth.2251.

Shah P, Gilchrist MA 2011. Explaining complex codon usage patterns with selection for translational efficiency, mutation bias, and genetic drift. Proceedings of the National Academy of Sciences of the United States of America 108:10231-10236. DOI: 10.1073/pnas.1016719108.

Shields DC, Sharp PM, Higgins DG, Wright F 1988. "Silent" sites in Drosophila genes are not neutral: evidence of selection among synonymous codons. Molecular Biology and Evolution 5:704-716.

Wang B, Shao Z-Q, Xu Y, Liu J, Liu Y, Hang Y-Y, Chen J-Q 2011. Optimal codon identities in bacteria: implications from the conflicting results of two different methods. PloS one 6:e22714. DOI: 10.1371/journal.pone.0022714.

Whittle CA, Extavour CG 2015. Codon and Amino Acid Usage Are Shaped by Selection Across Divergent Model Organisms of the Pancrustacea. G3 (Bethesda, Md.) 5:2307-2321. DOI: 10.1534/g3.115.021402.

Wu X, Jörnvall H, Berndt KD, Oppermann U 2004. Codon optimization reveals critical factors for high level expression of two rare codon genes in Escherichia coli: RNA stability and secondary structure but not tRNA abundance. Biochemical and Biophysical Research Communications 313:89-96. DOI: 10.1016/j.bbrc.2003.11.091.

Yang J-R, Chen X, Zhang J 2014. Codon-by-Codon Modulation of Translational Speed and Accuracy Via mRNA Folding. PLoS Biology 12:e1001910-14. DOI: 10.1371/journal.pbio.1001910.

Zhou T, Weems M, Wilke CO 2009. Translationally optimal codons associate with structurally sensitive sites in proteins. Molecular Biology and Evolution 26:1571-1580. DOI: 10.1093/molbev/msp070. 


\section{Figure 1}

The corseq pipeline

The corseq pipeline. (a) Population of the "peptide set"; selected proteins (suitable for the analysed species) are subjected to a kmer analysis using a sliding window approach, thus producing a set of amino acid kmers. (b) Identification of the pseudo-ORFs; RNAseq reads are sampled at each position by using a sliding window approach; the produced subsequences are translated and, if stop codons are not present, the corresponding pseudo protein is compared with the set of previously identified amino acid kmers. (c) Quantification of the nucleotide kmers generated by the pseudo-ORFs; sequences for which the corresponding protein matched an amino acid kmer, are split in their kmers that are thus quantified. (d) favoured codons computation; high and low frequency kmers are considered as proxy of high and low expression genes respectively; favoured codons are then computed by comparing, for each amino acid, the usage of the synonymous codons between these two sequences datasets. 


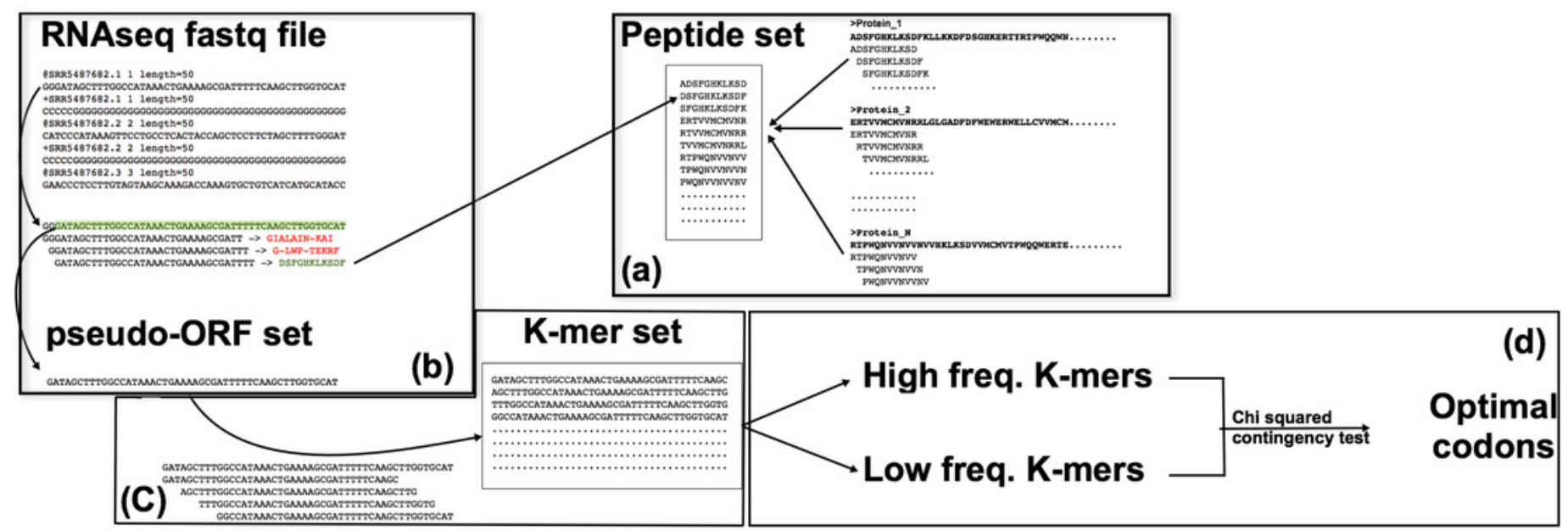




\section{Figure 2}

Consistency among replicates

Venn diagrams showing the number of detected favoured codons for 5 replicates, 4 values of number of bases and 5 species. For each presented diagram, each colour region reports the number of codons that were found to be favoured following a corseq run. Overlapping regions reports the number of concordant favoured codons between 5 replicates for the specified combination of species / number of bases. 

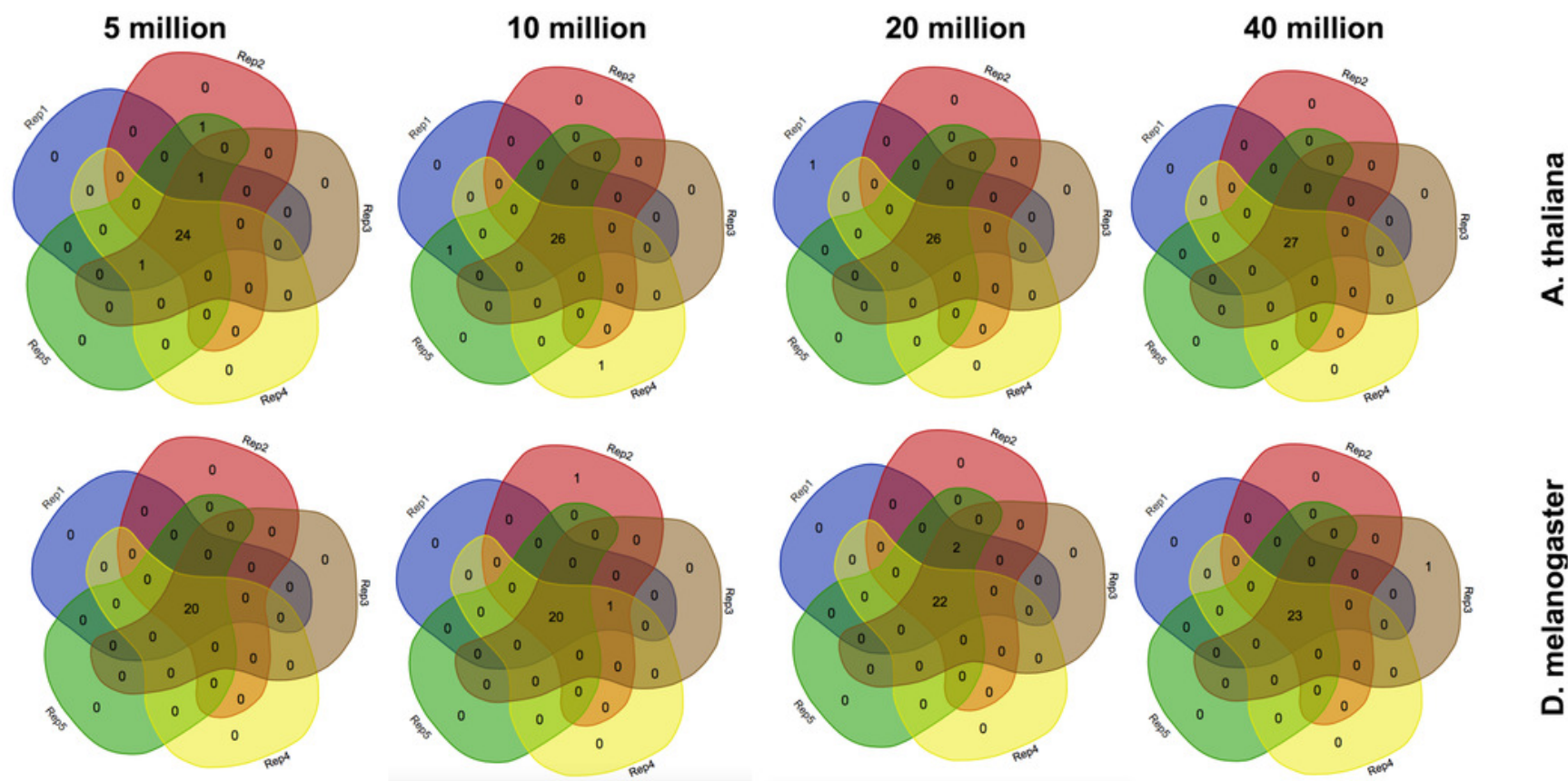

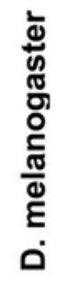
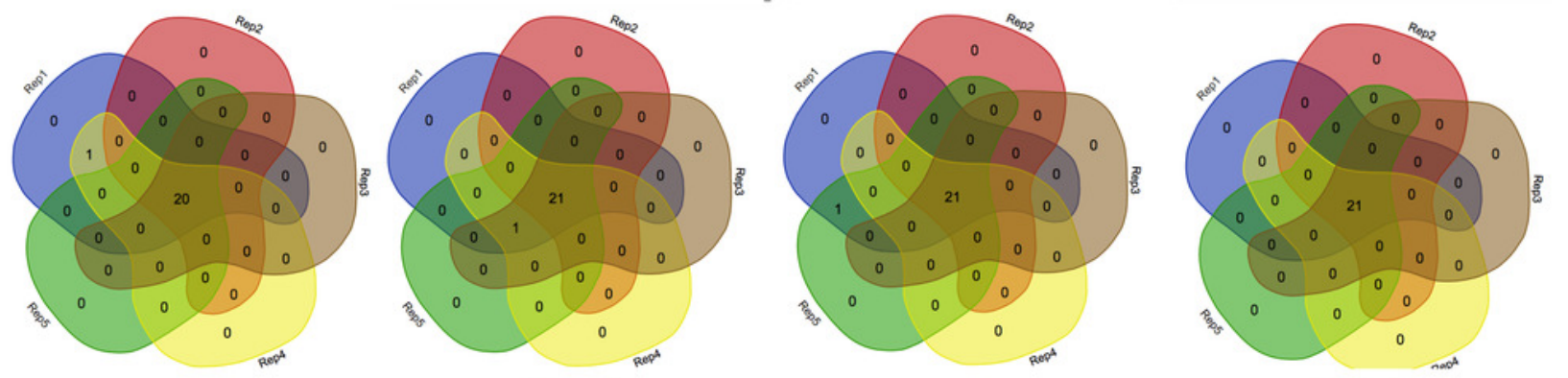

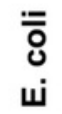
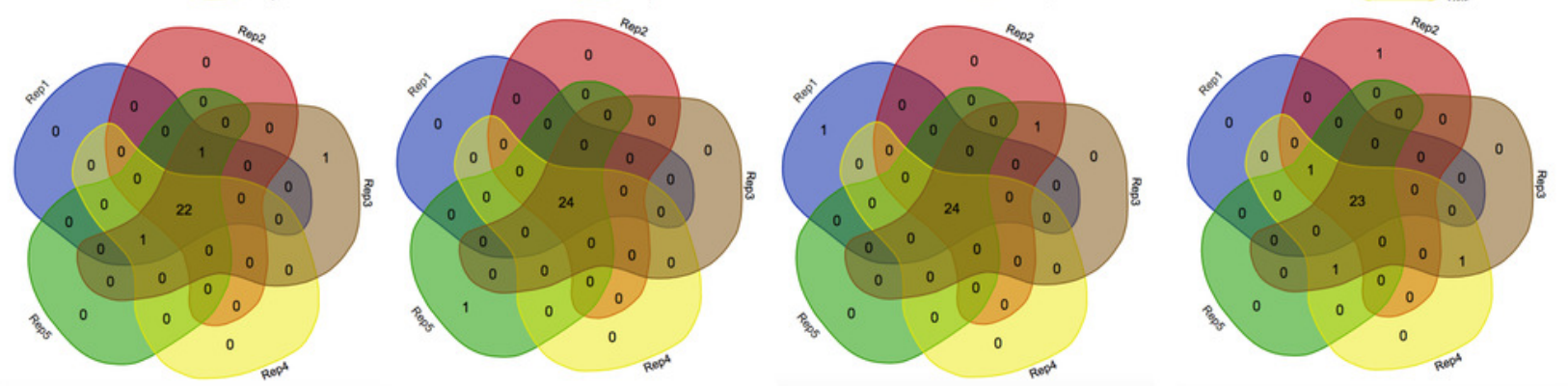

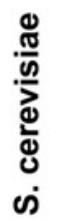
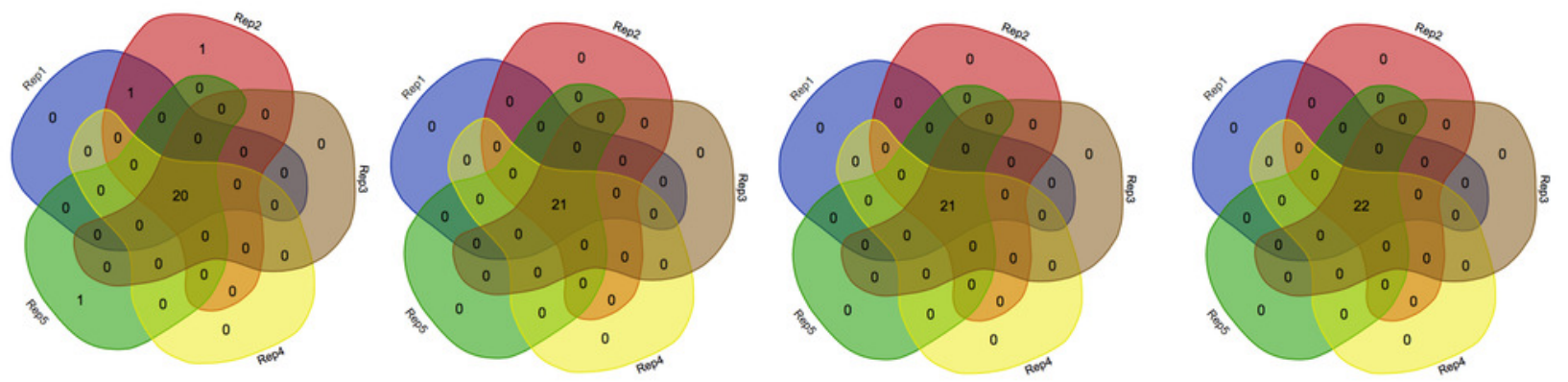

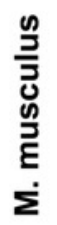




\section{Figure 3}

The effect of the kmer size in the detection of favoured codons

Venn diagrams showing the number of detected favoured codons for 5 replicates, 4 nucleotide kmer sizes and 5 species. For each presented diagram, each colour region reports the number of codons that were found to be favoured following a corseq run. Overlapping regions reports the number of concordant favoured codons between 5 replicates for the specified combination of species / nucleotide kmer size. 

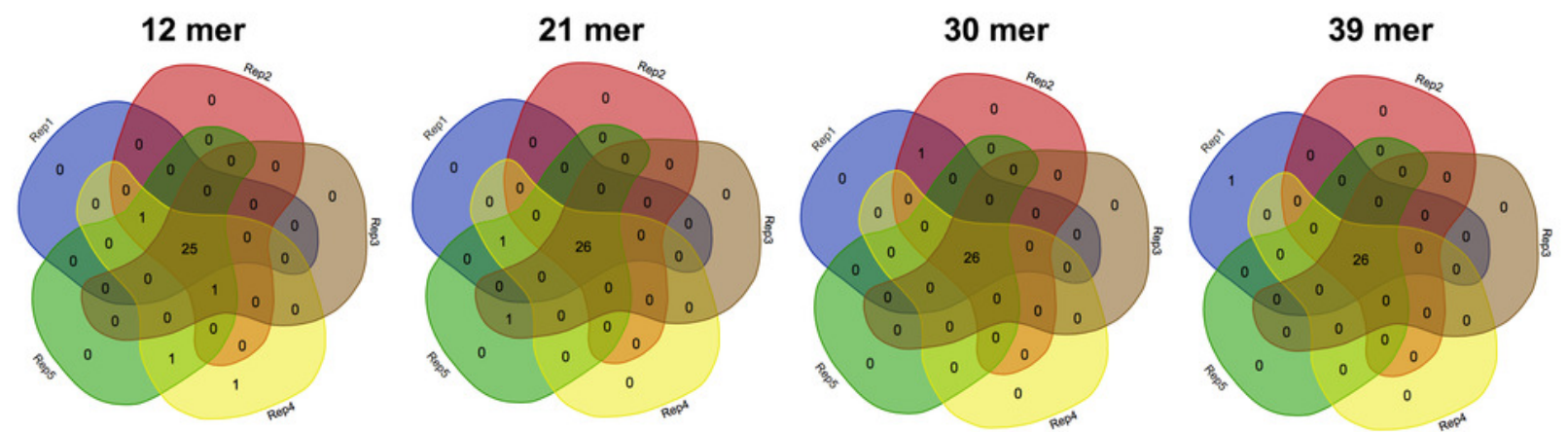

$\frac{\pi}{\frac{0}{\sigma}}$
$\frac{0}{\pi}$
$\frac{5}{5}$
$\dot{4}$
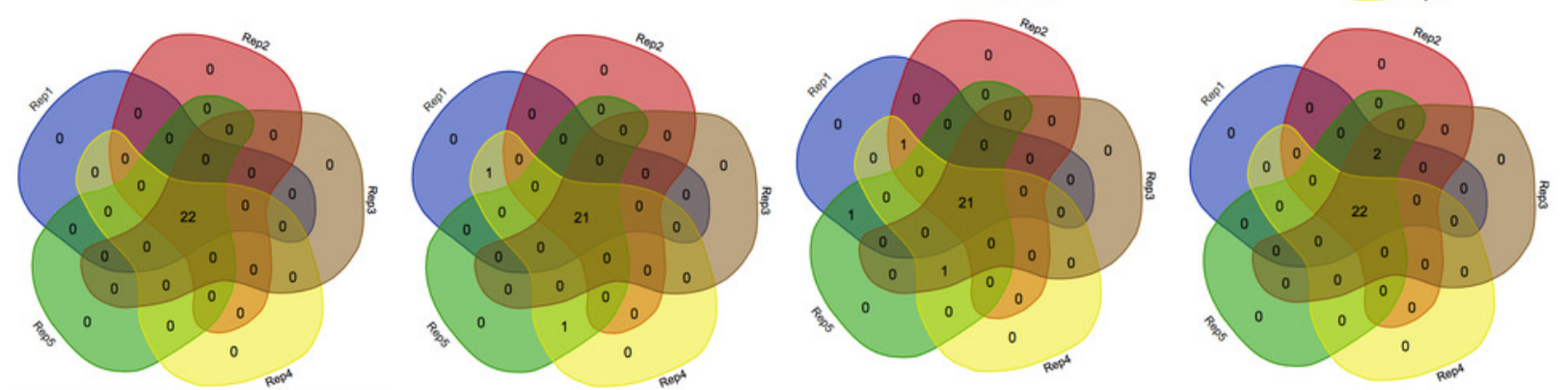

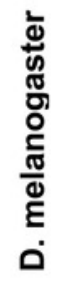
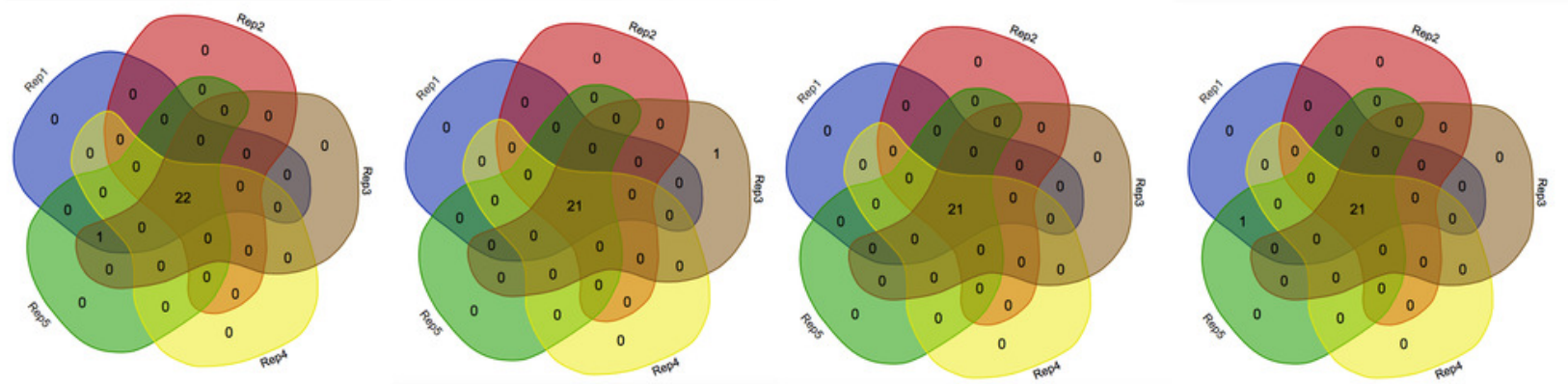

ن
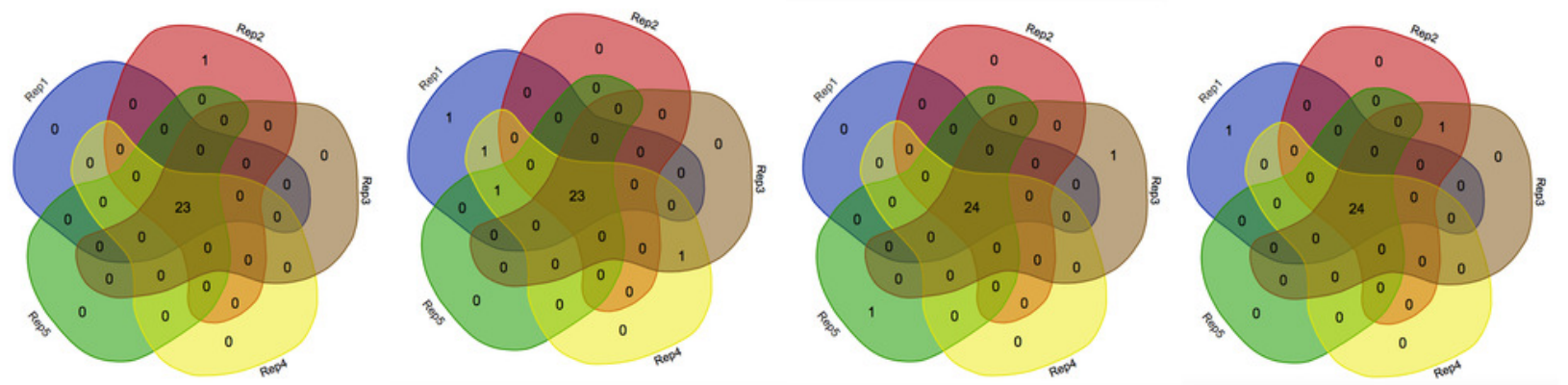

๗)
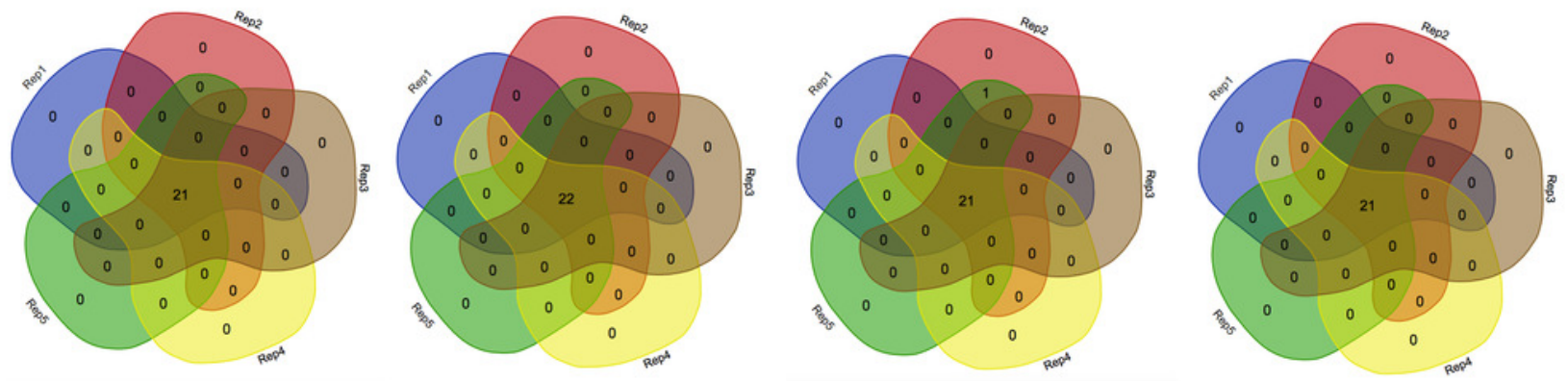

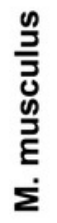




\section{Figure 4}

The effect of the kmer size on the corseq/CEM comparison

Venn diagrams showing the number of detected favoured codons that are shared between corseq with 4 different k-mer sizes and the conventional expression method. For each presented diagram, each colour region reports the number of codons that were found to be favoured following a corseq run. Overlapping regions reports the number of concordant favoured codons between 4 replicates and the conventional expression method for the specified species.
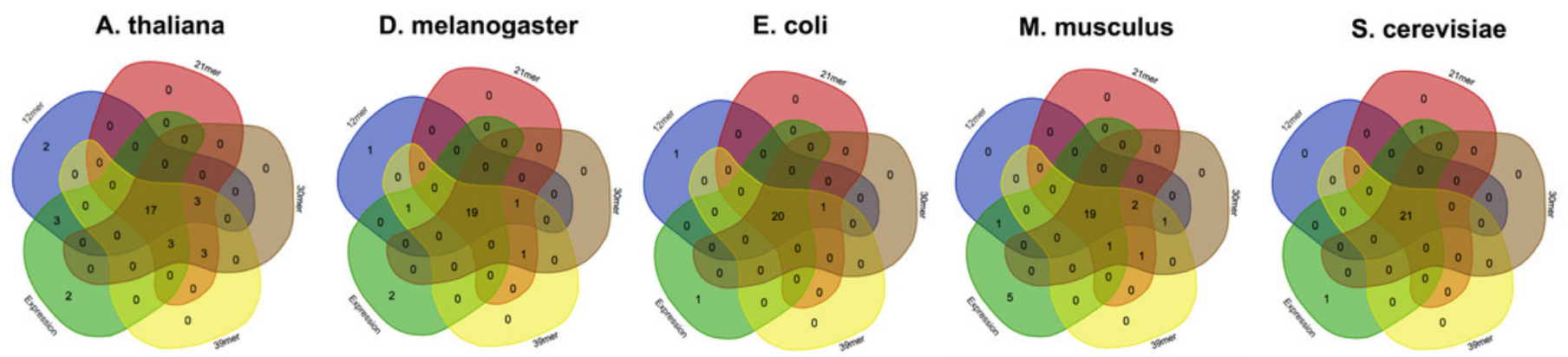


\section{Figure 5}

The effect of the initial dataset size on the favoured codons detection and running time

(a) Pearson correlation between the codons odds ratio calculated with corseq and the classical expression method. For each point the corseq odds ratio values were calculated starting from RNAseq dataset of different size. (b) Computational times of the corseq elaboration for RNAseq dataset of different size.
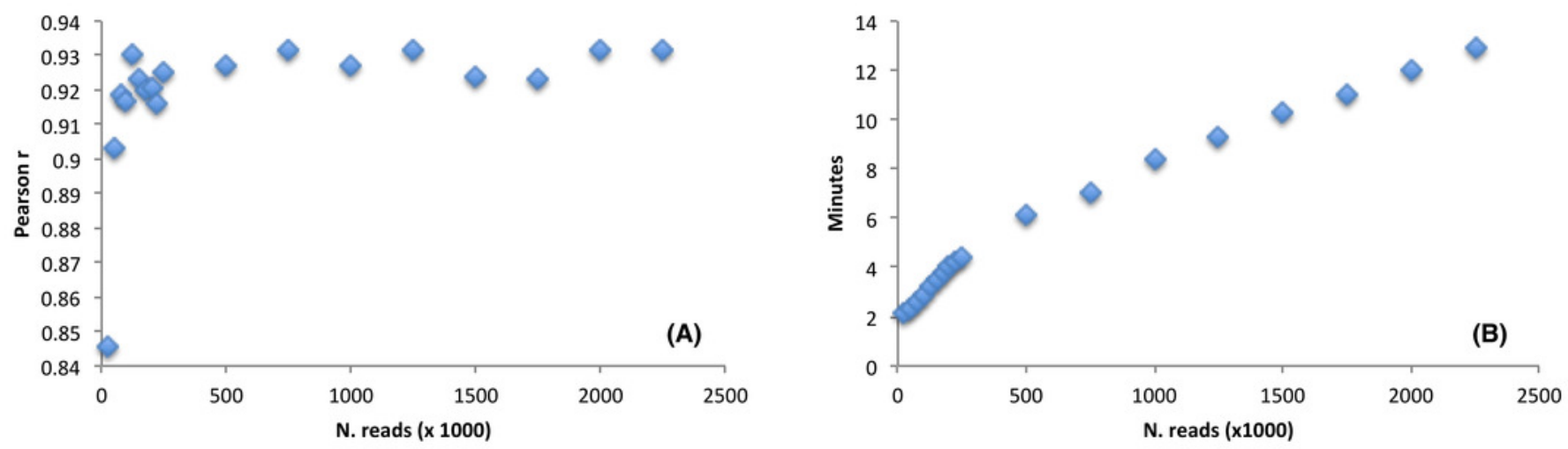


\section{Table $\mathbf{1}$ (on next page)}

The analyzed species

Expression and sequence data used to identify the favoured codons with the classical expression method. ${ }^{1}$ The NCBI SRA database is available at www.ncbi.nlm.nih.gov/sra/ . ${ }^{2}$ Phytozome 11 is available at http://genome.jgi.doe.gov/pages/dynamicOrganismDownload.jsf?organism=Phytozome, EMBL databases are available at http://fungi.ensembl.org/index.html , http://bacteria.ensembl.org/index.html , http://metazoa.ensembl.org/index.html . 


\begin{tabular}{lll}
\hline Species & RNAseq (SRA database) & Coding sequences $^{\mathbf{2}}$ \\
\hline Arabidopsis thaliana & SRR5487682 & Phytozome 11 \\
Oryza sativa & SRR5560727 & Phytozome 11 \\
Escherichia coli & SRR1796826 & EMBL bacteria \\
Staphylococcus aureus & SRR5456207 & EMBL bacteria \\
Lactobacillus pentosus & SRR2538344 & EMBL bacteria \\
Bacillus thuringiensis & SRR1296010 & EMBL bacteria \\
Saccharomyces cerevisiae & SRR5060294 & EMBL fungi \\
Candida albicans & SRR5444919 & EMBL fungi \\
Fusarium graminearum & SRR2170110 & EMBL fungi \\
Mus musculus & SRR5252256 & EMBL metazoan \\
Drosophila melanogaster & SRR3659025 & EMBL metazoan \\
Caenorhabditis elegans & SRR5312445 & EMBL metazoan \\
\hline
\end{tabular}

1 Table1: Expression and sequence data used to identify the optimal codons with the classical expression method. ${ }^{1}$

2 The NCBI SRA database is available at www.ncbi.nlm.nih.gov/sra/. ${ }^{2}$ Phytozome 11 is available at

3 http://genome.jgi.doe.gov/pages/dynamicOrganismDownload.jsf?organism=Phytozome, EMBL databases are

4 available at http://fungi.ensembl.org/index.html, http://bacteria.ensembl.org/index.html,

5 http://metazoa.ensembl.org/index.html. 


\section{Table 2 (on next page)}

Result of the corseq benchmark

Corseq benchmark results. The table reports the number of favoured codons that are commonly detected by both corseq and the CEM, the number of favoured codons only detected by corseq, those only detected only by CEM, the percentage of favoured codons sharing the same odds ratio direction (e.g. either $>1$ or $<1$ ) in the two methods, the Pearson correlation $r$ between the odds ratio calculated with the two methods (all correlations are significant with $p<0.05$ ) and the percentage of amino acid consensus (AAC) that is the ratio of amino acids for which at least one of the identified favoured codons is shared between the two methods. 


\begin{tabular}{lllllll}
\hline & Common & $\begin{array}{l}\text { corseq } \\
\text { specific }\end{array}$ & $\begin{array}{l}\text { CEM } \\
\text { specific }\end{array}$ & $\begin{array}{l}\text { Same odd ratio } \\
\text { direction (\%) }\end{array}$ & r & AAC(\%) \\
\hline A. thaliana & 21 & 6 & 4 & 0.86 & 0.93 & 0.89 \\
O. sativa & 23 & 2 & 3 & 0.92 & 0.95 & 1 \\
E. coli & 21 & 1 & 0 & 0.98 & 0.99 & 0.94 \\
S. aureus & 14 & 4 & 4 & 0.90 & 0.72 & 0.76 \\
L. pentosus & 15 & 5 & 6 & 0.86 & 0.82 & 0.88 \\
B. thuringenesis & 16 & 3 & 2 & 0.93 & 0.87 & 1.00 \\
& & & & & & \\
S. cerevisiae & 21 & 0 & 2 & 0.97 & 0.9 & 1 \\
F. graminearum & 17 & 4 & 8 & 0.78 & 0.72 & 0.83 \\
C. albicans & 20 & 1 & 3 & 0.97 & 0.94 & 0.94 \\
& & & & & & \\
M. musculus & 21 & 4 & 5 & 0.83 & 0.72 & 1 \\
D. melanogaster & 20 & 4 & 2 & 0.95 & 0.92 & 1 \\
C. elegans & 20 & 3 & 1 & 0.98 & 0.75 & 0.89 \\
\hline
\end{tabular}

Table 2: Corseq benchmark results. The table reports the number of optimal codons that are commonly detected by both corseq and the CEM, the number of optimal codons only detected by corseq, those only detected only by CEM, the percentage of optimal codons sharing the same odds ratio direction (e.g. either $>1$ or $<1$ ) in the two methods, the Pearson correlation $r$ between the odds ratio calculated with the two methods (all correlations are significant with $\mathrm{p}<0.05$ ) and the percentage of amino acid consensus (AAC) that is the ratio of amino acids for which at least one of the identified optimal codons is shared between the two methods. 